\title{
A STABLE CLASS OF SPACETIMES WITH NAKED SINGULARITIES
}

\author{
MARCUS KRIELE \\ Technische Universität Berlin, Fachbereich Mathematik \\ Sekr. MA 8-3, Straße des 17. Juni 136, 10623 Berlin, Germany \\ E-mail:kriele@sfb288.math.tu-berlin.de
}

\begin{abstract}
We present a stable class of spacetimes which satisfy the conditions of the singularity theorem of Hawking \& Penrose (1970), and which contain naked singularities. This offers counterexamples to a geometric version of the strong cosmic censorship hypothesis.

1. Introduction. One of the outstanding pursuits in relativity is to understand the nature of singularities. Evidence for the existence of regions with diverging curvature is supported by two classes of results.

1. The incompleteness theorems of Penrose, Hawking and others established the existence of inextensible, incomplete causal geodesics in physically realistic spacetimes (Hawking \& Ellis 1973, Beem \& Ehrlich 1981). These theorems can be applied to spacetimes resembling our universe on a cosmological scale or to those spacetimes containing dense, compact objects which are modelled by the existence of closed trapped surfaces. The most natural physical explanation is that curvature singularities develop in spacetime, thus preventing some of the causal geodesics from being complete. Mathematically however, the incompleteness theorems do not contain much information about the character of these singularities. In fact, as the Taub-Nut-spacetime exemplifies, it is possible for a locally completely regular region of spacetime to contain inextensible, incomplete causal geodesics.

2. Many exact solutions have regions of diverging curvature which contain incomplete, inextensible timelike geodesics. These exact solutions have high symmetry groups and it is not known to what extent they represent the generic case.

While the incompleteness theorems indicate the existence of singularities, it is the exact solutions which give us an idea of what they should look like. In particular, a survey of exact solutions indicates that in general, singularities which form in our future
\end{abstract}

International Classification for Physics: 04.20.Cv.

The paper is in final form and no version of it will be published elsewhere. 
are black holes rather than naked singularities. In the absence of other data this reasoning is highly plausible, but it is by no means clear how accurately our models represent generic singularities.

The nature of singularities, and especially the question of whether they are naked or resemble black holes, is essential for questions concerning the global predictability of the future from initial data. With the exception of the big bang, if all other singularities are (locally invisible) black holes, then they cannot influence the future of a partial Cauchy surface. On the other hand, if naked singularities exist, one would have to provide additional (singular) initial data at these singularities. ${ }^{1}$ Therefore their future would not be predictable using the regular initial data for a partial Cauchy surface located in their past. In part, this motivates the strong cosmic censorship hypothesis which is generally considered to be one of the most important problems in relativity. Penrose conjectured that for "physically realistic matter models" spacetime metrics which fail to be globally hyperbolic are "unstable". Unfortunately, neither the requirement of a "physically realistic matter model" nor the notion of "stability" are well defined. Two broad schools of thought exist on the approach to strong cosmic censorship. In the PDE-based approach a "physically realistic matter model" (such as vacuum) is specified. PDE-based strong cosmic censorship holds if for "almost all" initial data sets given at a partial Cauchy surface, the maximal globally hyperbolic solution of Einstein's equation is in fact locally inextensible. The alternative is a geometric approach in which certain energy, causality, and additional geometric conditions are imposed. Geometric strong cosmic censorship holds if "almost all" such inextensible spacetimes are globally hyperbolic. In this case one may also impose further conditions to guarantee that the singularities considered are due to incompleteness theorems.

The PDE-based approach has the advantage that the Whitney $C^{k}$ - and Sobolev topologies are reasonable for the initial data sets of a given spacelike hypersurface. It is however not realistic that any general theorem along these lines can be proven in the near future. Even proving the stability of Minkowski spacetime is highly complicated (Christodoulou \& Klainerman 1992). Further the requirement of a fixed matter model is restrictive to a non-generic class of spacetimes. Hence even if stability has been proven with respect to some matter model it is not clear that "perturbed" matter models will have the same qualitative properties. In this paper, we will therefore investigate a geometric version of the cosmic censorship hypothesis. The aim is to present stable counterexamples to strong cosmic censorship.

Let $\operatorname{Lor}(M)$ be the space of Lorentzian metrics on $M$ and fix a topology $\tau$ for $\operatorname{Lor}(M)$ which will be used to express the stability of examples. (Later we will specify several topologies $\tau$ for Lor $(M)$.) Since the incompleteness theorems are the main reason for expecting the existence of singularities, only those singularities predicted by these the-

\footnotetext{
${ }^{1}$ In the simplest case, the additional data could simply consist of stipulating that the singularity does not eject matter qualitatively different from the matter anticipated at the partial Cauchy surface. For instance, in (Kriele 1996) we have shown that the Cauchy problem for hypersurface-orthogonal $(2+1)$-dimensional dust spacetimes is well posed even though timelike singularities develop.
} 


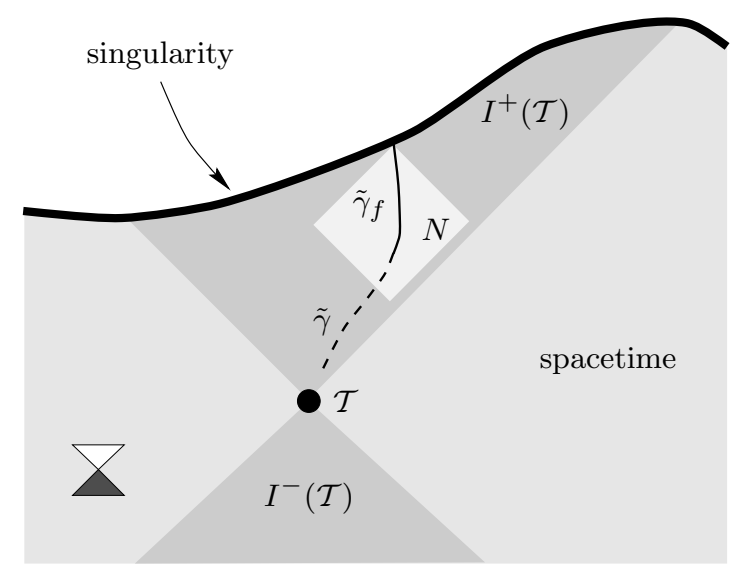

Figure 1: An example where strong cosmic censorship is satisfied. The set $N$ is a globally hyperbolic neighbourhood of the future endpiece $\tilde{\gamma}_{f}$ of the timelike geodesic $\tilde{\gamma}$.

orems are of physical concern. With this in mind, the following conjecture captures the essence of geometric strong cosmic censorship.

Conjecture 1 (geometric strong cosmic censorship). Let $(M, g)$ be a Lorentzian manifold and assume that

(i) $(M, g)$ is chronological,

(ii) the strong energy condition holds,

(iii) the dominant energy condition holds,

(iv) there exists a closed trapped surface $\mathcal{T}$.

Then in each $\tau$-neighbourhood $\mathcal{U}$ of $g$ in $\operatorname{Lor}(M)$, there is a metric $\tilde{g}$ satisfying (i)-(iii) such that each future inextensible, incomplete causal geodesic $\tilde{\gamma} \subset I^{+}(\mathcal{T})$ has a future endpiece $\tilde{\gamma}_{f}$ which is contained in some globally hyperbolic set.

If conditions (i), (ii), (iv) are satisfied and in addition we have the genericity condition, then the theorem of Hawking \& Penrose (1970) implies the existence of a causal incomplete geodesic $\gamma$ in $I^{+}(\mathcal{T}) \cup I^{-}(\mathcal{T})$. It would be natural to require the existence of a metric $\tilde{g}$ satisfying (i)-(iii) such that $(M, \tilde{g})$ is globally hyperbolic in each $\tau$-neighbourhood $\mathcal{U}$ of $g$ in $\operatorname{Lor}(M)$. However, this would be a global requirement whereas the restriction to singularities produced by closed trapped surfaces would be quasi-local. Hence conjecture 1 only requires that $(M, \tilde{g})$ is globally hyperbolic near the singularities (cf. Figure 1). It is physically plausible to expect $\gamma$ to be future incomplete and to be located in $I^{+}(\mathcal{T})$. Condition (iii) restricts the possible matter models to physically reasonable ones. Clearly, the validity of the conjecture depends crucially on the chosen topology $\tau$. In section 2 we will give an example of a spacetime which satisfies the dominant energy condition, contains a closed trapped surface, and has a timelike (in a well defined sense) singularity. In section 3 we will show that this is a counterexample to conjecture 1, when taken with respect to several topologies for $\operatorname{Lor}(M)$. 
2. Examples. In the following we concentrate on $(2+1)$-dimensional spacetimes which can be easily extended to obtain $(3+1)$-dimensional examples (cf. remark 2 below).

We need to recall the construction and some properties of irrotational 3-dimensional dust spacetimes $(\hat{M}, \hat{g})$ (for details see (Kriele 1996)). Let $u$ be the spacetime velocity of the dust particles and for any vector $v$ denote the 1 -form $\hat{g}(v, \cdot)$ by $v^{b} \cdot(\hat{M}, \hat{g})$ is an irrotational dust spacetime if $\mathrm{d} u^{b} \wedge u^{b}=0$ and in addition we have a function $\epsilon$ such that the energy momentum tensor reads $\hat{T}=\epsilon u^{b} \otimes u^{b}$. It is possible to find (essentially unique) coordinates $(t, x, y)$ such that the metric $\hat{g}$ is given by

$$
\hat{g}=-\mathrm{d} t^{2}+\left(V_{0}(x, y)+t q(x, y)\right)^{2} \mathrm{~d} x^{2}+W_{0}^{2}(x, y)\left(1+t\left(\frac{\partial V_{0}(x, y)}{\partial y}\right)^{-1} \frac{\partial q(x, y)}{\partial y}\right)^{2} \mathrm{~d} y^{2}
$$

where $V_{0}, W_{0}$ are arbitrary functions and $q$ is a solution of the linear PDE

$$
\frac{\partial^{2} q}{\partial x \partial y}+\left(W_{0}^{-1} \frac{\partial W_{0}}{\partial x}-\left(\frac{\partial V_{0}}{\partial y}\right)^{-1} \frac{\partial^{2} V_{0}}{\partial x \partial y}\right) \frac{\partial q}{\partial y}-\left(V_{0}^{-1} \frac{\partial V_{0}}{\partial y} W_{0}^{-1} \frac{\partial W_{0}}{\partial x}\right) q=0 .
$$

Denote the second fundamental form of the spacelike hypersurface $\{t=0\}$ by $k(X, Y)=$ $-g\left(\nabla_{X} Y, \partial_{t}\right)$ and observe that the principal curvatures of the hypersurface $\{t=0\}$ are given by $k_{1}=q / V_{0}$ and $k_{2}=\partial_{y} q / \partial_{y} V_{0}$. For any $p \in\{t=0\}$ let $k_{-}(p)=\min \left(k_{1}(p), k_{2}(p)\right)$ and $k_{+}(p)=\max \left(k_{1}(p), k_{2}(p)\right)$. The world line of the dust particle through $p$ ends in a curvature singularity

- at finite proper times $\frac{-1}{k_{-}(p)}, \frac{-1}{k_{+}(p)}$ if $k_{+}(p)>0>k_{-}(p)$,

- at finite proper time $\frac{-1}{k_{-}(p)}$ if $k_{-}(p)>0$,

- and at finite proper time $\frac{-1}{k_{+}(p)}$ if $k_{+}(p)<0$.

There are no other singularities. Since the light cones degenerate at the singularities, it is easy to see that geodesics ending in these singularities are future incomplete and do not lie in any globally hyperbolic subset. All singularities are weak in the sense that for all open sets $U$ with bounded volume, $\operatorname{vol}(U)=\int_{U} \sqrt{\operatorname{det}\left(g_{a b}\right)} \mathrm{d} t \wedge \mathrm{d} x \wedge \mathrm{d} y<\infty$, the spacetime average of the energy density,

$$
\frac{1}{\operatorname{vol}(U)} \int_{U} \epsilon(t, x, y) \sqrt{\operatorname{det}\left(g_{a b}\right)} \mathrm{d} t \wedge \mathrm{d} x \wedge \mathrm{d} y,
$$

is also bounded. In fact, this follows immediately since $\epsilon$ is given by

$$
\epsilon(t, x, y, z)=\frac{E(x, y)}{\left(V_{0}+t q\right)\left(\frac{\partial V_{0}}{\partial y}+t \frac{\partial q}{\partial y}\right)},
$$

where

$$
\begin{aligned}
E(x, y)= & \frac{\partial q}{\partial y} q+\left(\frac{\partial V_{0}}{\partial y}\right)^{2} \frac{\partial W_{0}}{\partial y} W_{0}^{-3}-\frac{\partial V_{0}}{\partial y} \frac{\partial^{2} V_{0}}{\partial y^{2}} W_{0}^{-2} \\
& -\frac{\partial V_{0}}{\partial y} \frac{\partial^{2} W_{0}}{\partial x^{2}} V_{0}^{-1} W_{0}^{-1}+\frac{\partial V_{0}}{\partial y} \frac{\partial W_{0}}{\partial x} \frac{\partial V_{0}}{\partial x} V_{0}^{-2} W_{0}^{-1}
\end{aligned}
$$

A specialization of these spacetimes will provide us with counterexamples to conjecture 1 . Let $\hat{M}=\mathbb{R} \times S^{1} \times \mathbb{R}$ where $x \equiv x+1$ is a cyclic coordinate. It is immediate from 


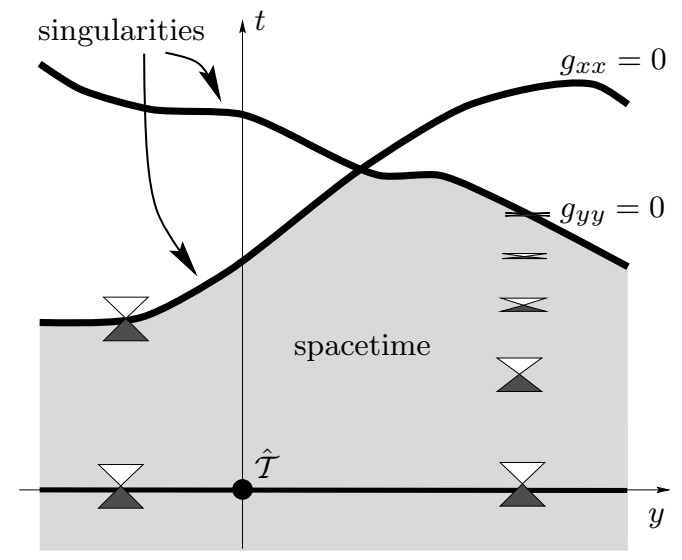

Figure 2: The singularity structure of $(\hat{M}, \hat{g})$. The light cone is degenerated in $y$-directions at $g_{y y}=0$ and degenerated in $x$-direction at $g_{x x}=0$. Both singularities are locally naked.

equation (2) that any pair of smooth, $x$-independent functions $\left(y \mapsto V_{0}(y), y \mapsto q(y)\right)$ determines a solution with $W_{0} \equiv 1$. We will choose these functions such that the compact submanifold $\hat{\mathcal{T}}=\{p \in \hat{M} \mid y=t=0\}$ is a closed trapped surface. Choose two linearly independent, future directed, null vector fields along $\hat{\mathcal{T}}$ which are orthogonal to $\hat{\mathcal{T}}$ and whose orthogonal projections to $\{t=0\}$ result in unit vectors. Denote the corresponding null expansions by $\theta^{ \pm} . \hat{\mathcal{T}}$ is a closed trapped surface if both, $\theta^{-}$and $\theta^{+}$are negative everywhere on $\hat{\mathcal{T}}$. The second fundamental form of $\hat{\mathcal{T}}$ (considered as a submanifold of the spacelike hypersurface $\{t=0\})$ is then given by $S(\xi, \eta)=-g\left({ }^{\{t=0\}} \nabla_{\xi} \eta, V_{0}^{-1} \partial_{y}\right)$. A straight forward calculation yields

$$
\theta^{ \pm}=\operatorname{tr}_{\{t=0\}}(k)-V_{0}^{-2} k\left(\partial_{y}, \partial_{y}\right) \mp \operatorname{tr}_{\hat{\mathcal{T}}}(S)
$$

and it follows immediately that $\hat{\mathcal{T}}$ is a closed trapped surface if $V_{0}, \partial_{y} V_{0}$ are positive and $q, q_{y}$ sufficiently negative at $\hat{\mathcal{T}}$. Observe that $E(x, y)=q \partial_{y} q-\partial_{y} V_{0} \partial_{y} \partial_{y} V_{0}$ can be guaranteed to be positive if $V_{0}$ and $q$ are suitably chosen. These spacetimes $(\hat{M}, \hat{g})$ satisfy assumptions (i)-(iv) in conjecture 1 and and in addition we know that no future endpiece $\gamma_{f}$ of any future inextensible, incomplete geodesic $\gamma$ lies in any globally hyperbolic subset.

Re mark 2. Each solution $(\hat{M}, \hat{g})$ determines a 4-dimensional spacetime $(M, g)$ which can be determined by setting $M=\hat{M} \times S^{1}$ and $g=\hat{g}+\mathrm{d} z^{2}$ where $z$ is a standard coordinate of $S^{1}$. Since $\hat{g}$ is a dust metric, the energy density and principal pressures of the 4 -dimensional spacetime $(M, g)$ are given by $\epsilon$ and $0,0,-\epsilon$, respectively. This implies that $(M, g)$ also satisfies assumptions (i)-(iv) of conjecture 1 . In addition, no future endpiece of any future inextensible, incomplete geodesic lies in any globally hyperbolic subset.

3. Several candidates for the topology $\tau$ of $\operatorname{Lor}(M)$. The discussions in this section are equally applicable to both the 4-dimensional example $(M, g)$ and to the 3dimensional example $(\hat{M}, \hat{g})$. For definiteness, we will refer only to $(M, g)$. 
It is clear that the validity of conjecture 1 depends crucially on the chosen topology $\tau$. For instance, if $\tau$ is the ultra-coarse topology $\tau_{\text {coarse }}=\{\operatorname{Lor}(M), \emptyset\}$ then the conjecture holds trivially since there exist globally hyperbolic spacetimes satisfying the energy conditions. On the other hand conjecture 1 does not hold if $\tau$ is the ultra-fine topology in which all subsets of $\operatorname{Lor}(\hat{M})$ are open. A physically meaningful topology should be invariantly defined and should have the property that differentiable perturbations (with compact support) are continuous. This guarantees that our intuitive notion of "being close" is respected by this topology. At first glance, two families of topologies seem to be especially natural, the Whitney $C^{k}$-topologies (Lerner 1973) and the compact-open $C^{k}$-topologies for $k \geq 0$.

3.1. The Whitney $C^{k}$-topology, $\tau_{\text {Whit }}^{k}$. The Whitney $C^{k}$-topologies are defined as follows (Beem \& Ehrlich 1981, p. 28). Let $\left(U_{\ell}, \varphi_{\ell}\right)_{\ell \in \mathbb{N}}$ be a countable fixed atlas such that each compact subset of $M$ intersects only finitely many of the $U_{l}$. A neighbourhood of $h \in \operatorname{Lor}(M)$ is then given by the set $\mathcal{U}(h)$ of all metrics $\tilde{h}$ such that

$$
\sum_{\substack{0 \leq l \leq|\alpha| \leq k \\ 1 \leq i_{1} \leq \ldots \leq i_{l} \leq \operatorname{dim}(M) \\ 1 \leq a, b \leq \operatorname{dim}(M)}}\left|\frac{\partial^{|\alpha|}}{\partial x_{i_{1}}{ }^{\alpha^{i_{1}}} \ldots \partial x_{i_{l}}{ }^{\alpha^{i_{l}}}}\left(\left(\varphi_{\ell}^{*} \tilde{h}\right)_{a b}-\left(\varphi_{\ell}^{*} h\right)_{a b}\right)\right|_{x}<\eta_{\ell}(x) \quad \forall \ell \in \mathbb{N}
$$

where $\eta_{\ell}: U_{\ell} \rightarrow \mathbb{R}^{+} \backslash\{0\}$ are continuous functions. These open sets form a sub-basis of the Whitney $C^{k}$-topology which is independent of the atlas chosen.

Proposition 3. Conjecture 1 does not hold with respect to the Whitney $C^{k}$-topology $\tau_{\text {Whit }}^{k}$.

Pr o of. Without loss of generality we can restrict our attention to the case where $M$ is an open submanifold of a smooth manifold $\bar{M}$ and is covered by a single chart which extends to a neighbourhood of $M \cup \partial M$ in $\bar{M}$. Choose compact subsets $K_{n}(n \in \mathbb{N})$ with $K_{n} \subset \operatorname{int}\left(K_{n+1}\right), \cup_{n=1}^{\infty} K_{n}=M$ and choose $\eta: M \rightarrow \mathbb{R}^{+}$such that $\eta(p)<1 / n$ for all $p \in M \backslash K_{n}$. Let

$$
\mathcal{U}=\left\{h \in \operatorname{Lor}(\hat{M})\left|\sum_{1 \leq a, b \leq \operatorname{dim}(M)}\right|\left(\varphi^{*} g\right)_{a b}-\left.\left(\varphi^{*} h\right)_{a b}\right|_{x}<\eta(x)\right\}
$$

It follows that each $h \in \mathcal{U}$ has a continuous extension to $\bar{M}$ and is degenerate at $\partial M$. Further, this singular set is timelike and therefore conjecture 1 does not hold for any $\tau_{\text {Whit }}^{k}$.

One possible objection to the use of the Whitney $C^{k}$-topologies may be that these topologies allow for arbitrarily good control of the metric near the singularities.

3.2. The compact-open $C^{k}$-topology, $\tau_{\mathrm{c}-\mathrm{o}}^{k}$. The compact-open $C^{k}$-topology is another intrinsically defined topology. A sub-basis $\{\mathcal{U}(h,(U, \varphi), K, n)\}$ is determined by a collection of symmetric tensor fields $h$, charts $(U, \varphi)$, compact subsets $K \subset U$ and natural numbers $n$. We have $\hat{h} \in\{\mathcal{U}(h,(U, \varphi), K, n)\}$ if and only if 


$$
\sum_{\substack{0 \leq l \leq|\alpha| \leq k \\ 1 \leq i_{1} \leq \ldots \leq i_{l} \leq \operatorname{dim}(M) \\ 1 \leq a, b \leq \operatorname{dim}(M)}}\left|\frac{\partial^{|\alpha|}}{\partial x_{i_{1}}{ }^{i^{i_{1}}} \ldots \partial x_{i_{l}} \alpha^{i_{l}}}\left(\left(\varphi^{*} \tilde{h}\right)_{a b}-\left(\varphi^{*} h\right)_{a b}\right)\right|_{x}<1 / n
$$

for all $x \in K$. This topology is invariantly defined but has the disadvantage that it does not allow for any control near the singularity. To see this note that any neighbourhood of $h$ contains the intersection of finitely many sets $\left\{\mathcal{U}\left(h_{l},\left(U_{l}, \varphi_{l}\right), K_{l}, n_{l}\right)\right\}_{l=1, \ldots \ell}$ and that this can only restrict the metric in the compact subset $K_{1} \cup \ldots \cup K_{\ell}$. Such a topology is too coarse since with respect to this topology there are metrics with diverging curvature in any neighbourhood of Minkowski half space.

ExAmple 4 . Let $M=\{(t, x, y, z) \mid y<0\}$ and let

$$
f_{n}(y)= \begin{cases}1 & \text { for } y \leq-\frac{1}{\ln (n)} \\ 1-n \mathrm{e}^{\frac{-1}{y+1 / \ln (n)}} & \text { for } y>-\frac{1}{\ln (n)}\end{cases}
$$

It is easy to see that for each neighbourhood of the standard Minkowski metric there is an $n_{0} \in \mathbb{N}$ such that for all $n \geq n_{0}$ the metric $g_{n}=-\mathrm{d} t^{2}+f_{n}(y) \mathrm{d} x^{2}+\mathrm{d} y^{2}+\mathrm{d} z^{2}$ is contained in this neighbourhood. Further all $g_{n}$ satisfy the strong and the dominant energy conditions and have a curvature singularity at $y=0$.

3.3. An intermediate $C^{k}$-topology $\tau_{\text {int }}^{k}$ for a subset of metrics. The idea is to define a topology analogous to the Whitney $C^{k}$-topology where the functions $\eta_{\ell}$ are replaced by constants. This would make the new topology much coarser near the singularity. By naively replacing $\eta_{\ell}$ by constants we would obtain a topology which depends on the system of charts $\left(U_{l}, \varphi_{l}\right)$. Recall that $M$ is a manifold with boundary $\partial M$ which can be identified with the singularities. We demand that $\left\{\left(U_{\ell}, \varphi_{\ell}\right)\right\}$ represents a countable atlas for the manifold with boundary such that for each $p \in M \cup \partial M$ there are only finitely many $U_{l}$ which cover $p$. We can now replace the function $\eta_{\ell}$ by constants arriving at a well defined topology $\tau_{\text {int }}^{k}$ for Lor $(M)$. Observe that in each neighbourhood of $g$ there are metrics which can be smoothly extended beyond $\partial M$ as Lorentzian metrics. Hence with respect to this topology, the singularity itself does not appear to be stable. This is due to the fact that the topology measures "closeness" in terms of symmetric bilinear forms and their derivatives and not in terms of curvature. In fact, the energy density of $g$ diverges while the energy density of those "nearby" bilinear forms is bounded. We could add $C^{0}$-conditions to curvature invariants such as the energy density $\epsilon$. It is less of a restriction to consider only the set $\operatorname{Lor}_{\partial M}(M)$ of those bilinear forms $h$ such that, given any open set $U \subset M \cup \partial M, h_{\mid U \cap M}$ cannot be locally extended to $\partial M \cap U$ as a Lorentzian metric.

Proposition 5. Conjecture 1 does not hold with respect to the intermediate $C^{k}$ topology $\tau_{\text {int }}^{k}$ of $\operatorname{Lor}_{\partial M}(M)$.

Pro of. The singularity is fixed and (with respect to any continuous metric extension) timelike. The claim follows since "timelike" is a stable property with respect to the intermediate $C^{k}$-topologies. 

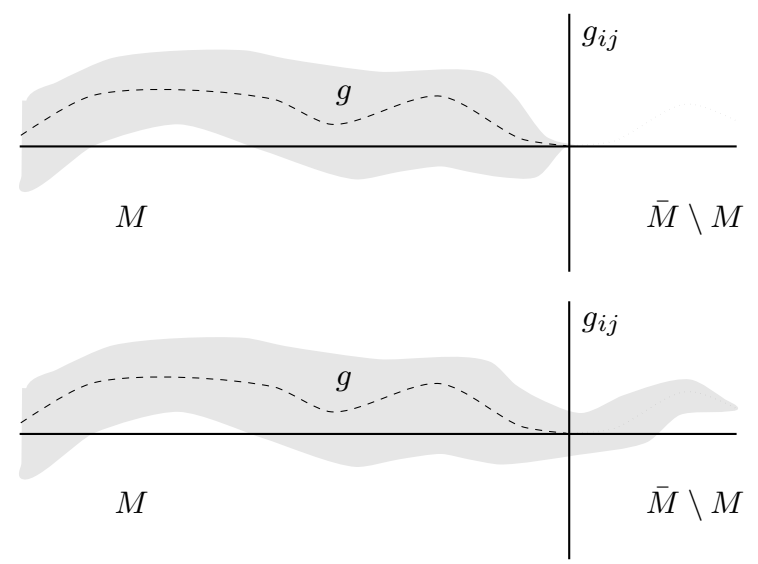

Figure 3: A typical neighbourhood of the metric $g$ in a Whitney topology (section 3.1) and a typical neighbourhood with respect to the topology $\tau(U)$ introduced in section 3.4

An objection may be that our topologies do not allow for the singularity to be shifted. While the concept of a fixed singularity is chart dependent, in what follows we will give an example of a topology where the location of the singularity can be shifted with respect to a fixed coordinate system.

3.4. A topology $\tau(U)$ where the singular set is not fixed. Observe that $(M, g)$ is isometrically embedded into a generalized pseudo Riemannian manifold $(\bar{M}, \bar{g})$, where $\bar{M}$ is a manifold and $\bar{g}$ a smooth symmetric $\left(\begin{array}{l}0 \\ 2\end{array}\right)$-tensor field. $\partial M \subset \bar{M}$ consists of the set of points where $\bar{g}$ is degenerate. Let $U$ be a neighbourhood of $M \cup \partial M$ in $\bar{M}$ and consider the set $S_{2}(U)$ of all smooth, symmetric $\left(\begin{array}{l}0 \\ 2\end{array}\right)$-tensor fields on $U$ together with the corresponding Whitney $C^{2}$-topology. Let $p \in M$ be a fixed point and $\operatorname{Lor}_{p}(U) \subset S_{2}(U)$ be the subspace of all bilinear forms on $U$ which are Lorentzian in a neighbourhood of $p$. For each $h \in \operatorname{Lor}_{p}(U)$ we can assign the connected component $U(p, h)$ of $\{q \in U \mid h$ is Lorentzian at $q\}$ which contains the point $p$. Denote the restriction of $h$ to $U(p, h)$ by $f_{p}(h)$. We can now equip $f_{p}\left(\operatorname{Lor}_{p}(U)\right)$ with the identification topology $\tau(U)$ induced by $f_{p}$. Observe that $f_{p}(h)$ contains the set of all Lorentzian metrics on $M$ which can be smoothly extended to $U$ as bilinear forms. Through this somewhat roundabout method we have defined a topology for which the location of the singularity is no longer fixed (by an open neighbourhood).

Let $U_{n} \subset \bar{M}$ be a sequence of open sets $U_{n+1} \subset U_{n}$ such that $M \cup \partial M \subset U_{n}$ and $\bigcap_{n=1}^{\infty} U_{n}=M \cup \partial M$. Denote by $\operatorname{Dom}_{p}\left(U_{n}\right)$ the set of all $g \in f_{p}\left(\operatorname{Lor}_{p}\left(U_{n}\right)\right)$ for which the dominant energy condition holds. The following lemma implies that all metrics close to $g$ satisfying the dominant energy condition have a singularity close to the singularity of the original metric.

Lemma 6. For each $n \in \mathbb{N}$ there exists a neighbourhood $\mathcal{U}_{n}$ of $g$ such that all metrics in $\mathcal{U}_{n} \cap \operatorname{Dom}_{p}\left(U_{n}\right)$ are inextensible as Lorentzian metrics. Further, the neighbourhoods $\mathcal{U}_{n}$ can be chosen such that $\mathcal{U}_{n+1} \cap \operatorname{Dom}_{p}\left(U_{n+1}\right) \subset i^{*}\left(\mathcal{U}_{n} \cap \operatorname{Dom}_{p}\left(U_{n}\right)\right)$ for all $n$, where $i: U_{n+1} \rightarrow U_{n}$ is the canonical injection. 
Pr o of. Let $\eta_{0}$ be a positive function on $U_{1}$. We will inductively construct a sequence of positive functions $\left\{\eta_{n}\right\}_{n \in \mathbb{N}}$ which defines a sequence of open sets $\left\{\mathcal{U}_{n}\right\}_{n \in \mathbb{N}}$ as in equation (6) (with $\eta_{\ell}$ being replaced by $\eta_{n}$ ). Choose a neighbourhood $V_{n}$ of $U_{n+1}$ such that its closure is contained in $U_{n}$ and denote by $\partial V_{n}$ its boundary in $U_{n}$. Since $\epsilon_{\mid \partial V_{n}}$ is strictly negative, we can find a function $\eta_{n}<\eta_{n-1}$ such that for all $h \in \mathcal{U}_{n}$ the vector $\partial_{t}$ is timelike and $T\left(\partial_{t}, \partial_{t}\right)_{\mid \partial V_{n}}$ is negative. Consequently, no $f_{p}(h) \in \mathcal{U}_{n} \cap \operatorname{Dom}_{p}(U)$ can be extended to $\partial V_{n}$. The second assertion follows since $\left\{\eta_{n}\right\}$ is a monotonically decreasing sequence of functions.

Lemma 6 would fail without the dominant energy condition.

Proposition 7. Conjecture 1 does not hold with respect to any topology $\tau\left(U_{n}\right)$.

Proof. Let $p \in \partial M$ and observe that the degenerate $\operatorname{subspace}_{\operatorname{Rad}}(g)=\{v \in$ $\left.T_{p} \bar{M} \mid g(v, \cdot)=0\right\}$ is transverse to $T_{p} \partial M$. Since this property is stable with respect to the Whitney $C^{2}$-topology associated with $U_{1}$, each $\mathcal{U}_{n}$ can be chosen such that it holds for all $h \in \mathcal{U}_{n}$. By Lemmas 13 and 14 in (Kriele \& Lim 1995) the divergence of the energy density is also stable with respect to this topology. Kriele \& Lim (1995) have shown that any Lorentzian metric which admits a smooth extension as a bilinear form and satisfies the transverseness condition on Rad must have a timelike singularity, provided the dominant energy condition holds and the energy density diverges. Consequently, conjecture 1 is violated with respect to the topology $\tau\left(U_{n}\right)$ of $f_{p}\left(\operatorname{Lor}_{p}\left(U_{n}\right)\right)$.

Observe that the topologies $\tau(U)$ (and also the intermediate topologies $\tau_{\text {int }}^{k}$ in section 3.3) are very coarse. Within each neighbourhood of $g$ one can find Lorentzian metrics for which the energy density diverges one order of magnitude faster than the energy density of $g$ (Kriele \& Lim 1995, proposition 15). In the same neighbourhood there are also other metrics whose energy density does not diverge at all (however the latter violate the dominant energy condition). Hence stability with respect to these topologies is a very strong requirement.

4. Conclusion. We have shown that in general, even when stability is accounted for, the singularities produced by the incompleteness theorem of Hawking and Penrose (Hawking \& Ellis 1973, Theorem 2) do not satisfy strong cosmic censorship. For this it was crucial that (unlike lightlike shell focusing singularities) our singularities are timelike. Our aim has been to construct simple counterexamples, but in doing so we have obtained properties which may be considered artificial. These properties are summarized by the following.

- The closed trapped surfaces in the 4-dimensional examples have topology $\mathbb{T}^{2}$ rather than $S^{2}$. This comes from a construction which is essentially 3-dimensional.

- Since we used (3-dimensional) dust solutions for the construction of counterexamples, our singularities are not very strong. In fact, the energy density exists as a spacetime average and they also fail to be strong in the sense of Tipler (1977) or Królak (1987). The idea of "strong curvature singularities" was physically motivated by the expectation that gravitational collapse (as suggested by the incompleteness theorems) would result in strong curvature singularities. Our examples arise in exac- 
tly this way but contain only weak singularities, therefore this expectation cannot be maintained. If our (weak) singularities are regarded as physically non-singular, we can obtain examples of physically non-singular spacetimes satisfying the assumptions of the incompleteness theorem of Hawking \& Penrose. Consequently, the physical interpretation of the incompleteness theorems would have to be revised. On the other hand, it is promising to see that by perturbing our spacetimes we can obtain strong, naked singularities which still preserve the dominant energy condition.

- The singularities studied here require the energy density to dominate one of the principal pressures by an order of the least one. This is always the case for singularities which can be modelled by smooth bilinear forms (Kriele \& Lim 1995, Theorem 21). It is therefore conceivable that counterexamples can be avoided by adding the assumption that the quotient of any two eigenvalues of $T_{b}^{a}$ is bounded. In the absence of this, it would be necessary to resort to the hyperbolic nature of Einstein's equations.

What we have learnt from the examples presented here is that stability and the energy conditions alone are not sufficient to ensure cosmic censorship — more detailed assumptions on the matter model must be made.

Acknowledgements. I thank Giselle Lim for numerous discussions.

\section{References}

J. K. Beem and Ehrlich (1981), Global Lorentzian geometry, Marcel Dekker, New York and Basel.

D. Christodoulou and S. Klainerman (1992), The Global Nonlinear Stability of Minkowski Space, Princeton University Press, Princeton.

S. W. Hawking and G. F. R. Ellis (1973), The large scale structure of space-time, Cambridge University Press, Cambridge.

S. W. Hawking and R. Penrose (1970), 'The singularities of gravitational collapse and cosmology', Proc. Roy. Soc. Lond. A 314, 529-548.

M. Kriele (1996), 'Shell singularities of dust spacetimes', Class. Quantum Grav., to appear.

M. Kriele and G. Lim (1995), 'Physical properties of geometrical singularities', Class. Quantum Grav. 12, 3019-3035.

A. Królak (1987), 'Towards the proof of the cosmic censorship hypothesis in cosmological space-times', J. Math. Phys. 28(1), 138-141.

D. E. Lerner (1973), 'The space of Lorentz metrics', Commun. Math. Phys. 32, 19-38.

P. Musgrave, D. Pollney and K. Lake (1994), 'GRTensorII', The program and its documentation can be found at astro.queensu.ca in the directory /pub/grtensor. Queen's University, Kingston, Ontario, Canada.

F. Tipler (1977), 'Black holes in closed universes', Nature 270, 500-501. 